\title{
Effect of Esketamine Nasal Spray on Olfactory Function and Nasal Tolerability in Patients with Treatment-Resistant Depression: Results from Four Multicenter, Randomized, Double-Blind, Placebo-Controlled, Phase III Studies
}

\author{
Richard L. Doty ${ }^{1} \cdot$ Vanina Popova $^{2}$ (1) - Crystal Wylie ${ }^{1}$ Maggie Fedgchin ${ }^{3} \cdot$ Ella Daly $^{4} \cdot$ Adam Janik $^{5}$. \\ Rachel Ochs-Ross ${ }^{3} \cdot$ Rosanne Lane $^{3} \cdot$ Pilar Lim $^{3} \cdot$ Kim Cooper $^{6} \cdot$ Rama Melkote $^{3} \cdot$ Carol Jamieson $^{7} \cdot$ Jaskaran Singh $^{5,8}$. \\ Wayne C. Drevets ${ }^{5}$
}

Accepted: 4 May 2021 / Published online: 7 July 2021

(c) The Author(s) 2021

\begin{abstract}
Background Intranasal drug delivery offers a non-invasive and convenient dosing option for patients and physicians, especially for conditions requiring chronic/repeated-treatment administration. However, in some cases such delivery may be harmful to nasal and olfactory epithelia.

Objective The aim of this study was to assess the potential impact of long-term intermittent treatment with esketamine nasal spray, taken in conjunction with an oral antidepressant (AD), on olfactory function and nasal tolerability in patients with treatment-resistant depression (TRD).

Methods A total of 1142 patients with TRD participated from four multicenter, randomized, double-blind, phase III studies: three short-term studies (two in patients aged 18-64 years, one in patients $\geq 65$ years), and one long-term maintenance study of esketamine nasal spray $+\mathrm{AD}$ versus placebo nasal spray $+\mathrm{AD}$. Across the four studies, assessments were performed at 208 sites in 21 countries. Olfactory function was measured using the 40-item University of Pennsylvania Smell Identification Test $\left(\right.$ UPSIT $^{\circledR}$ ) and the single-staircase Snap \& Sniff ${ }^{\circledR}$ Odor Detection Threshold Test (S\&S-T). Nasal tolerability, including nasal examinations and a quantitative, self-administered nasal symptom questionnaire (NSQ), was also assessed. Data were analyzed using analyses of covariance.

Results Of 1142 participants, 734 were women (64.3\%). The mean age of all participants ranged from 45.7 to 70.0 years across the studies. Overall, 855 patients received esketamine nasal spray $+\mathrm{AD}$ and 432 received placebo nasal spray + AD. Objective evaluation of nasal function showed no evidence of an adverse impact following esketamine administration. Based on the UPSIT ${ }^{\circledR}$ and S\&S-T results, intranasal administration of esketamine had no effect on the odor identification or threshold test scores compared with placebo nasal spray + oral AD. Similarly, repeated administration with esketamine nasal spray had no meaningful impact on assessments of nasal function. No dose-response relationship was observed between esketamine doses and the olfactory test scores. Esketamine nasal spray was well tolerated, as indicated by responses on the NSQ and negative nasal examination findings.

Conclusion Findings from this analysis indicate that there was no evidence of adverse effect on either olfactory or nasal health measures with repeated intermittent administration of esketamine nasal spray at any dose over the course of short-term (4 weeks) or long-term (16-100 weeks) studies.

Clinical trial registration TRANSFORM-1: NCT02417064, date of registration: 15/04/2015; TRANSFORM-2: NCT02418585, date of registration: 16/04/2015; TRANSFORM-3: NCT02422186, date of registration: 21/04/2015; SUSTAIN-1: NCT02493868, date of registration: 10/07/2015.
\end{abstract}

Extended author information available on the last page of the article 


\section{Key Points}

Intranasal drug delivery is an efficacious and convenient means for the treatment of numerous disorders.

However, long-term treatment with intranasal drugs has the potential to cause local toxicity, including anosmia, epistaxis, and numbness.

In this analysis, the potential impact of repeated intermittent dosing with intranasally administered esketamine nasal spray on olfactory function and nasal tolerability in patients with treatment-resistant depression was investigated.

No evidence of adverse effects of repeated administration of esketamine nasal spray on olfactory or nasal function were identified at any esketamine dose.

\section{Introduction}

Major depressive disorder (MDD), a highly recurrent condition, is a debilitating psychiatric illness that often requires long-term treatment. Despite the availability of numerous antidepressant therapies, nearly one-third of patients with MDD do not respond to available antidepressants and are considered to have treatment-resistant depression (TRD) $[1,2]$. While there is no consensus definition for TRD, it is typically considered present in individuals with MDD who have not responded to at least two different antidepressant treatments of adequate dose and duration during a current episode of depression [3].

Esketamine, the S-enantiomer of racemic ketamine and a N-methyl-D-aspartate receptor antagonist, is approved as a nasal spray in conjunction with an oral antidepressant by the United States Food and Drug Administration (FDA), European Medicines Agency (EMA), and other global health authorities for treatment of TRD in adults $[4,5]$. In addition, esketamine has recently received approval in the United States for the treatment of depressive symptoms in patients with MDD and acute suicidal ideation or behavior [6], as well as being approved by the EMA for the treatment of depressive symptoms in patients with MDD who are experiencing a psychiatric emergency.

It is well established that intranasal drug delivery can be an efficacious and convenient means for the treatment of numerous disorders, including migraine headaches and neuropsychiatric diseases, as it bypasses the effects of first-pass metabolism, gastrointestinal absorption, and potentially the blood-brain barrier (i.e., for the fraction that may reach the cribriform plate) $[7,8]$. In the case of esketamine, the wide plume angle produced by the nasal spray device delivers medication mainly to the respiratory epithelium, leading to uptake of drug into the bloodstream. The mean total absolute bioavailability of esketamine administered through this route is $\sim 48 \%$, which is the result of a fraction of the esketamine dose being absorbed directly from the nasal mucosa and the remainder of the dose being swallowed and subject to first pass metabolism. This is much higher than the bioavailability of esketamine observed with oral administration ( 8\%) [9].

That being said, intranasal drugs, especially those given as long-term treatment, have the potential for causing local toxicity, including altered smell function, epistaxis, and numbness, depending upon their active ingredients and excipient contents [10]. Damage to the sense of smell can be debilitating, as this sensory system significantly contributes to the flavor of foods and beverages and plays an important role in detecting environmental hazards such as fire, leaking natural gas, and toxic foodstuffs. Importantly, loss of smell, such as that caused by pollution, has been associated with the formation of aberrant proteins in central nervous system olfactory structures associated with the pathology of Alzheimer's and other neurodegenerative diseases, reflecting transport of nanoparticles and other materials into the brain via the receptor cells [11]. One intranasally administered drug, Zicam, an over-the-counter zinc-containing nasal spray, was shown to produce anosmia in some users, resulting in its withdrawal from the US market in 2009 by the FDA [12].

These issues have raised awareness regarding the possibility that intranasally administered drugs may impact the ability to smell. Thus, appropriate assessments have been implemented to evaluate whether this is the case for new products that involve intranasal delivery. As part of the phase III program in TRD, we investigated whether esketamine nasal spray demonstrated any potential to cause treatmentemergent alterations in smell function or other adverse nasal effects. Specifically, quantitative state-of-the-art measures of smell function, as well as detailed nasal examinations and a 13-item Nasal Symptom Questionnaire (NSQ), were conducted repeatedly throughout the course of the studies of the phase III program in TRD, at 208 sites in 21 countries.

\section{Methods}

\subsection{Participants and Study Design}

A total of 1142 patients with TRD from four phase III, double-blind (DB) registration studies in the esketamine TRD program were evaluated. Exclusion criteria prior to study entry included a score on the University of Pennsylvania Smell Identification Test (UPSIT ${ }^{\circledR}$ ) [13] suggestive 
of anosmia, as described later in this paper, and a history or presence of any anatomical or medical condition that, per the investigators' clinical judgment, might impede delivery or absorption of the intranasal study drug. Intranasally administered medications that contained decongestants (vasoconstrictors) were allowed during the treatment phases for nasal congestion, if required; however, their use within $1 \mathrm{~h}$ of administration of the nasal spray study medication (esketamine/placebo) was not permitted. Patients taking other intranasal drugs, such as steroids, were excluded.

Specific details of the TRD studies from which the data for the present analyses were obtained are presented elsewhere [14-17]. Briefly, patients with non-response to at least one prior antidepressant treatment, as measured by the Massachusetts General Hospital Antidepressant Treatment Response Questionnaire, and who were currently taking a different antidepressant were screened to allow for participation. During this 4-week period, response to the patients' current oral antidepressant treatment was assessed. Patients meeting study-defined criteria for TRD, including nonresponse to the current antidepressant treatment (defined as $\leq 25 \%$ improvement on the Montgomery-Asberg Depression Rating Scale [MADRS] from week 1 to week 4, and a MADRS total score $\geq 28$ at weeks 2 and 4) were enrolled in one of the short-term DB Induction (IND) studies that involved the administration of a newly initiated oral antidepressant in combination with either esketamine nasal spray or placebo nasal spray. The oral antidepressant was administered daily. It was initiated at the starting dose (e.g., duloxetine $30 \mathrm{mg}$, escitalopram $10 \mathrm{mg}$, sertraline $50 \mathrm{mg}$, or venlafaxine extended release $75 \mathrm{mg}$ ) and subsequently titrated to the maximal tolerated dose per label. Those patients in the short-term TRANSFORM-1 (ClinicalTrials.gov identifier NCT02417064) [15] and TRANSFORM-2 (NCT02418585) [16] studies, whose ages ranged from 18 to 64 years, were randomized to receive either esketamine $56 \mathrm{mg}$ or $84 \mathrm{mg}$ or placebo nasal spray twice weekly for 4 weeks. In TRANSFORM-1, the dose was fixed for a given patient, whereas in TRANSFORM-2, the dose regimen was flexible. Those in the short-term TRANSFORM-3 (NCT02422186) [17] study, which enrolled patients $\geq 65$ years of age, were randomized to esketamine or placebo nasal spray under a flexible dose regimen (a lower dose of $28 \mathrm{mg}$ was also included as a starting dose in addition to $56 \mathrm{mg}$ and $84 \mathrm{mg}$ ) (Fig. 1).

At the end of the IND period, responders (defined as $\geq$ $50 \%$ reduction MADRS total score from baseline) from the TRANSFORM-1 and TRANSFORM-2 studies were eligible to enter the optimization (OP) phase of the long-term relapse prevention study (SUSTAIN-1, NCT02493868) [14] (Fig. 1). At the end of the 12-week OP phase, patients in SUSTAIN-1 who met study-defined criteria for stable remission or stable response continued into a randomized withdrawal maintenance (MA) phase, in which patients previously treated with esketamine plus an oral antidepressant were randomly assigned $(1: 1)$ to either a group that continued their esketamine treatment or to a group that received a placebo nasal spray, while continuing the ongoing oral antidepressant.

At the end of TRANSFORM-3 (short-term study in patients $\geq 65$ years), eligible patients, regardless of response, had the opportunity to participate in an open-label, longterm study (SUSTAIN-2); however, because of the lack of a control group, that study did not include smell tests.

\subsection{Intranasal Treatment Administration}

Esketamine nasal spray was supplied as a solution containing $16.14 \%$ weight/volume (w/v) esketamine hydrochloride (equivalent to $14 \% \mathrm{w} / \mathrm{v}$ esketamine base) in a single-use nasal spray device that delivered two sprays (one spray per nostril) of esketamine $28 \mathrm{mg}$ in total. Citric acid monohydrate, disodium edetate, sodium hydroxide, and water were used as excipients. Intranasal placebo was supplied as a solution containing water with a bittering agent $(0.001 \mathrm{mg} / \mathrm{mL}$ denatonium benzoate) provided in matching nasal spray devices, to reduce the likelihood that patients may be functionally unblinded by the bitter taste of esketamine. Prior to starting treatment in the DB studies, participants received instructions on the appropriate use of the device including practicing spraying the device in the air using a placebo training nasal spray device.

During the short-term studies, esketamine or placebo nasal spray was self-administered at the clinical site under direct supervision twice a week for 4 weeks. For patients entering the OP phase of the SUSTAIN-1 study, from weeks 5 to 8 , the treatment session frequency was reduced to once weekly and thereafter every 2 weeks or once weekly to maintain remission/response. In addition, patients continued the oral antidepressant medication.

\subsection{Olfactory Function}

Each patient's smell function was assessed using two standardized olfactory tests, the UPSIT ${ }^{\circledR}[13,18]$ and the Snap \& Sniff ${ }^{\circledR}$ Odor Detection Threshold Test (S\&S-T) [19]. These tests, described in detail in the next sections, were selected based on their high reliability, validity, practicality, and extensive normative data ([13, 19-24]; for a discussion of the relative strengths and limitations of these tests, see [25]). For this multinational study, the UPSIT $^{\circledR}$ was translated into the relevant languages and some odorants and response alternative names were changed to ones more familiar in specific cultures. All translations were conducted by an international certified translation company that followed relevant industry standards for translation services (ISO 9001 and ISO 17100, as well as ISO 13485 and ISO 14910 standards). Test administrators at 


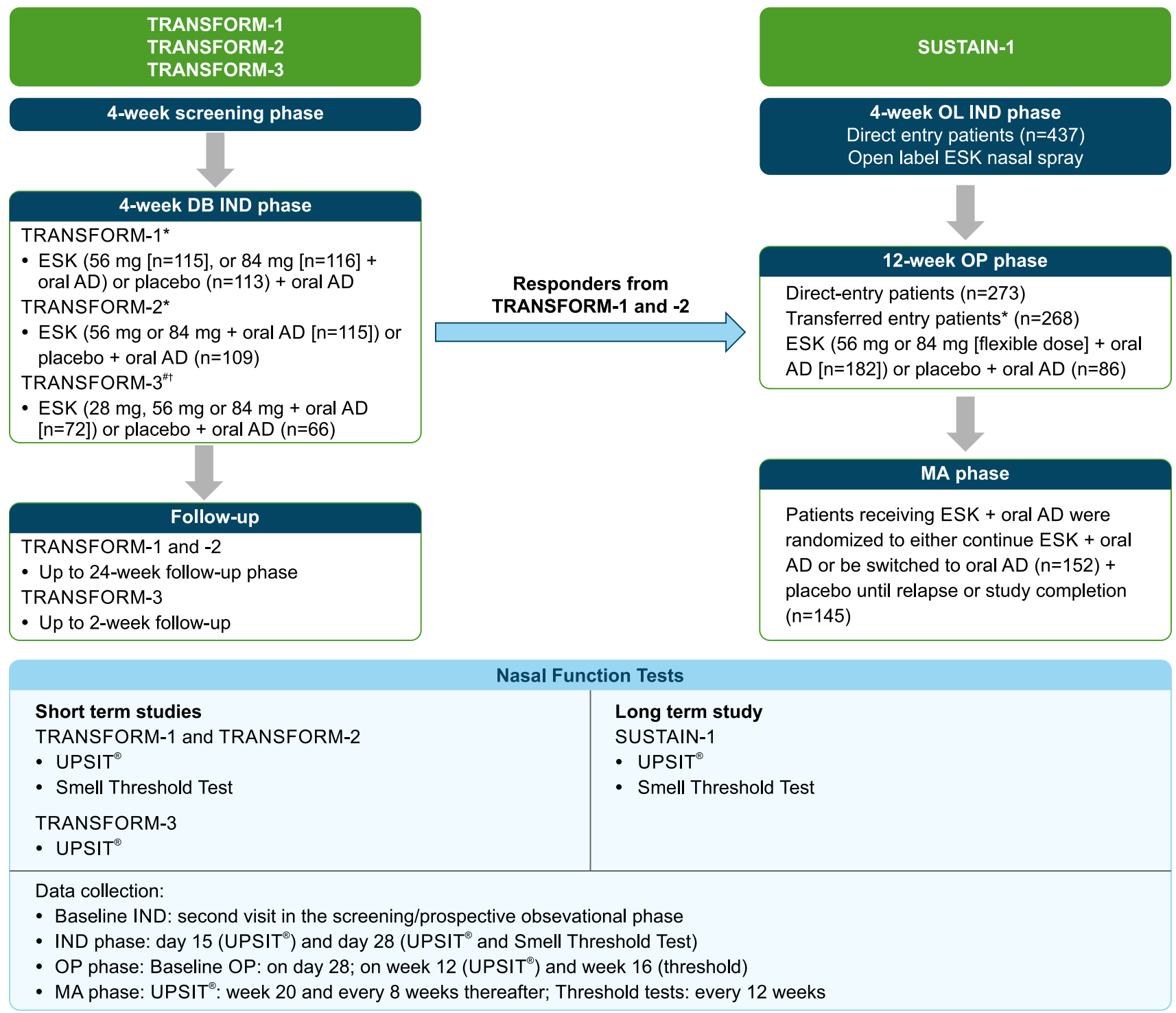

Fig. 1 Study designs and nasal function tests. *age 18-64 years, "age $\geq 65$ years; ${ }^{\dagger}$ responders from TRANSFORM-3 study did not enter in SUSTAIN-1 study. $A D$ antidepressant, $D B$ double-blind, ESK esketamine, $I N D$ induction, $M A$ maintenance, $O L$ open-label, $O P$ opti-

all clinical trial sites were trained to administer these tests, and testing occurred prior to dosing, with the UPSIT ${ }^{\circledR}$ being administered before the S\&S-T test. Both tests were performed bilaterally (i.e., both nostrils at the same time) for each patient.

\subsubsection{University of Pennsylvania Smell Identification Test (UPSIT ${ }^{\oplus)}$}

The UPSIT ${ }^{\circledR}$ quantitatively assesses a patient's ability to identify odors. This reliable (test-retest $r=0.95$ ) and wellvalidated olfactory test consists of four booklets, each booklet containing ten pages $[13,18,20]$. Located on each page is a $1.27 \mathrm{~cm} \times 2.54 \mathrm{~cm}$ label containing a microencapsulated mization, UPSIT ${ }^{\circledR}$ University of Pennsylvania Smell Identification Test. References: SUSTAIN-1 [14]; TRANSFORM-1 [15]; TRANSFORM-2 [16]; TRANSFORM-3 [17]

odorant. Each odorant is released by scratching the label in a standardized fashion. Four response choices (i.e., names) are provided for each odorant, and the patient is required to indicate which one best represents the released smell. A response is required even if no odor is perceived, that is, the test is forced-choice. A no-odor choice is not provided. The test score is calculated as the total number of correct responses out of 40 and, in this study, was presented as the percent correct responses (i.e., test score $/ 40 \times 100$ ). Since a response must be made on every test item, and there are four alternatives for each odorant, a person without a sense of smell would be expected to score, on average, at chance level (i.e., $25 \%$ or 10/40). There is an empirical sampling 
distribution around this value, such that anosmia is defined as a score from 6 to 18. Scores lower than this are highly improbable based on chance and are suggestive of the avoidance of the correct answer (i.e., malingering). UPSIT ${ }^{\circledR}$ testing occurred during screening to establish the patient's baseline sensitivity and throughout each treatment phase.

To simplify evaluation in the elderly population and reduce the time burden for completion of the test, only three booklets of the four-booklet UPSIT ${ }^{\circledR}$ were administered in TRANSFORM-3, i.e., 30 rather than 40 test items.

\subsubsection{Snap \& Sniff ${ }^{\oplus}$ Odor Detection Threshold Test (S\&S-T)}

The ability of the patients to detect very low odorant concentrations was measured using the S\&S-T, a test in which different concentrations of an odorant are presented using metal wands [19, 24]. The wands have a release system that briefly exposes an odorized tip for sampling. Half-log dilution steps of the odorant phenyl ethyl alcohol (PEA) in light USP-grade mineral oil ranging from $10^{-2}$ to $10^{-9} \mathrm{vol} / \mathrm{vol}$ are employed along with comparison non-odor diluent blanks.

The stimuli are presented using a single staircase psychophysical procedure in which the first trial begins at the $1 \times 10^{-6}$ concentration step. A trial consists of the successive presentation of one wand with an odorant and another with a non-odor blank diluent in a predetermined counterbalanced order. The patient is asked to indicate which wand produces a stronger smell, the first or the second. At low concentrations, a patient will perceive only the presence of something different from the blank diluent, not an odor quality (e.g., rose). The ability to perceive odor quality only occurs at higher concentrations. If no difference is perceived, an answer must still be given (i.e., the test is forced-choice). If an incorrect response occurs on any trial, the staircase is moved upward one full-log step. When a correct response is made on five consecutive trials at a given concentration, the staircase is reversed and subsequently moved up or down in $0.50 \mathrm{log}$ increments or decrements, depending upon the patient's performance on two pairs of trials at each concentration step. The test measure is the mean of the last four of seven staircase reversals, i.e., up to down transitions, in $\log _{10}$ concentration units.

\subsubsection{Times of Olfactory Testing}

The UPSIT ${ }^{\circledR}$ was administered in the three short-term studies (TRANSFORM-1, TRANSFORM-2, and TRANSFORM-3), and in the long-term relapse prevention study (SUSTAIN-1). The S\&S-T was performed in the TRANSFORM-1, TRANSFORM-2, and SUSTAIN-1 studies (Fig. 1). Of note, the S\&S-T was not performed in a shortterm study in older patients (TRANSFORM-3) following a protocol amendment implemented to reduce patient burden.
Prior to initiation of nasal spray medication, the UPSIT ${ }^{\circledR}$ and the S\&S-T were performed in the screening/prospective observational phase to establish the patient's baseline sensitivity. Subsequently, the UPSIT $^{\circledR}$ was administered on day 15 and both the UPSIT ${ }^{\circledR}$ and the S\&S-T on day 28 of the IND phase. The degree of change from baseline was subsequently determined. The percent change from baseline served as the dependent measure for each patient for each test. In TRANSFORM-3, after protocol amendment, the UPSIT $^{\circledR}$ was performed before the start of induction (prior to randomization) and at day 28 or the end of treatment.

In the relapse prevention study (SUSTAIN-1), the UPSIT $^{\circledR}$ was performed at week 12 of the OP phase and week 20 of the MA phase, and then every 8 weeks. The S\&S-T was performed at week 16 of the OP phase and every 12 weeks of the MA phase. A schedule of these tests is provided in Supplementary Table 1 in the electronic supplementary material [ESM].

\subsection{Nasal Tolerability}

\subsubsection{Nasal Examinations}

A nasal examination (including the upper respiratory tract/ throat) was conducted at screening, every 4 weeks before dosing throughout the treatment phases, and in the followup phase, by a qualified healthcare practitioner at each study site (Supplementary Table 1 in the ESM). The examination at screening was performed to rule out any patients with anatomical or medical conditions that may impede drug delivery or absorption. Subsequent examinations consisted of visual inspection of the nostrils, nasal mucosa, and throat for nasal erythema, rhinorrhea, rhinitis, capillary/blood vessel disruption, and epistaxis, and were graded as absent, mild, moderate, or severe.

\subsubsection{Nasal Symptom Questionnaire}

Nasal tolerability associated with intranasal administration was also assessed using a patient-reported questionnaire developed by the study sponsor (Supplementary Table 2 in the ESM). The NSQ is a 13-item questionnaire that assesses nasal symptoms, which were rated as none, mild, moderate, or severe, based on each patient's experience at the time of the assessment. Patients completed the NSQ both pre-dose and at $1 \mathrm{~h}$ post-dose weekly during the IND phase, weekly (if applicable based on treatment frequency) during the OP phase, and every other week during the MA phase (Supplementary Table 1 in the ESM). 


\subsection{Statistical Analyses}

Descriptive statistics of the UPSIT ${ }^{\circledR}$ score (calculated as percent of the items correctly identified) and percent changes from baseline were summarized at each scheduled time point during each treatment phase. Baseline IND assessments were used for computing change.

The S\&S-T score was calculated as the mean of the fourth through seventh staircase reversals (the last four up-down transitions) as recorded on the case report form. If all reversals were not completed, the data were not included in the analyses. A smell deficit was defined as thresholds above $-2.40 \log _{10} \mathrm{vol} / \mathrm{vol}$ [21]. Descriptive statistics for observed values and changes from baseline (IND) were summarized at each scheduled time point during each treatment phase.

Frequency distributions of ratings on the NSQ were generated for each of the items for each administration. Shift from pre-dose to post-dose assessment on each dosing day throughout the study were provided by treatment group to see if there was any change after administration of intranasal study medication. Frequency of patients who reported moderate or severe symptoms at any post-dose time point were summarized by treatment group. In addition, a listing of severe symptoms was also generated.

Statistical differences among the test scores of the different treatment groups were analyzed using post-hoc analyses of covariance (ANCOVAs).

\section{Results}

\subsection{Demographic and Baseline Characteristics}

A total of 1142 patients with TRD from the four phase III studies were evaluated in this study. Among these, 137 (12\%) patients were 65 years of age or older. The mean age of elderly patients was $70.0 \pm 4.52$ years. That of nonelderly patients ranged from 45.7 to 46.5 years across the remaining three studies. The majority of the patients were women (64.3\%), White (88.9\%), and were from the European region (Table 1). Other than age, race, and sex, there were no other notable differences observed in baseline characteristics across the studies.

Overall, 855 patients received intranasal esketamine + oral antidepressant for a median (range) duration of 57.0 (1-743) days and 432 received intranasal placebo + oral antidepressant for 26.0 (1-655) days, cumulatively across four studies. Of the total esketamine-exposed patients, 115 patients were exposed to a 56-mg dose for a median (range) duration of 92.0 (4-743) days, 116 were exposed to $84 \mathrm{mg}$ for $26(1-542)$ days, and 624 were exposed to a flexible dose of esketamine for 65.5 (1-692) days. Of the total, 145 participants who received esketamine during the IND and OP phases of TRANSFORM-1, TRANSFORM-2, and SUSTAIN-1 studies and subsequently received placebo in the MA phase of SUSTAIN-1 study, were included in both groups (esketamine and placebo) for exposure.

\subsection{Olfactory Function (UPSIT ${ }^{\circledR}$ and S\&S-T)}

The results of the three short-term DB efficacy studies with weekly dosing were similar to one another, as indicted below.

\subsubsection{TRANSFORM-1 (Fixed-Dose 4-Week Study; Twice Weekly Dosing)}

The mean percent changes in UPSIT ${ }^{\circledR}$ scores from screening during the DB IND phase in each treatment group (intranasal placebo + oral antidepressant; intranasal esketamine $56 \mathrm{mg}+$ oral antidepressant; intranasal esketamine $84 \mathrm{mg}+$ oral antidepressant) were unremarkable (Table 2).

Consistent with the UPSIT ${ }^{\circledR}$ percent changes, the mean S\&S-T scores did not show any noteworthy difference between esketamine/oral antidepressant and placebo/oral antidepressant treatment groups between the baseline and day-28 test sessions (Table 3 ). Occurrence of a small olfactory deficit (defined as a threshold $\geq-2.40$ ) was noted in 1 of 104 (1.0\%) patients in the intranasal esketamine $56 \mathrm{mg}+$ oral antidepressant treatment group, 1 of $100(1.0 \%)$ of those in the intranasal esketamine $84 \mathrm{mg}$ + oral antidepressant treatment group, and 2 of the 106 $(1.9 \%)$ patients in the intranasal placebo + oral antidepressant treatment group.

\subsubsection{TRANSFORM-2 (Flexible-Dosed 4-Week Study, Twice Weekly Dosing)}

Similar to the results obtained in the TRANSFORM-1 study, in TRANSFORM-2 there were no clinically noteworthy changes or percent changes in the UPSIT ${ }^{\circledR}$ scores between screening phase and the end of the IND phase (day 28) in either treatment group (Table 2). In total, 1 of $109(0.9 \%)$ patients treated with intranasal esketamine + oral antidepressant and 4 of $100(4.0 \%)$ patients treated with intranasal placebo + oral antidepressant had a smell threshold $\geq-2.40$ during the DB IND phase. As with the UPSIT ${ }^{\circledR}$, the S\&S-T scores (Table 3) did not appear to differ between patients treated with intranasal esketamine + oral antidepressant and those treated with intranasal placebo + oral antidepressant. 
Table 1 Baseline demographics of studies TRANSFORM-1 [15], TRANSFORM-2 [16], TRANSFORM-3 [17], and SUSTAIN-1 [14] (safety analysis set)

\begin{tabular}{|c|c|c|c|c|}
\hline Characteristic & $\begin{array}{l}\text { TRANSFORM-1 adult } \\
\text { patients }(N=344)\end{array}$ & $\begin{array}{l}\text { TRANSFORM-2 adult } \\
\text { patients }(N=224)\end{array}$ & $\begin{array}{l}\text { TRANSFORM-3 elderly } \\
\text { patients }(N=137)\end{array}$ & $\begin{array}{l}\text { SUSTAIN-1 direct } \\
\text { entry }(N=437)\end{array}$ \\
\hline \multicolumn{5}{|l|}{ Age, years } \\
\hline Mean (SD) & $46.3(11.16)$ & $45.7(11.89)$ & $70.0(4.52)$ & $46.5(11.0)$ \\
\hline Median (range) & $47.0(18-64)$ & $47.0(19-64)$ & $69.0(65-86)$ & $48.0(19-64)$ \\
\hline \multicolumn{5}{|l|}{ Age category, $n(\%)$} \\
\hline $18-44$ years & $139(40.4)$ & $94(42.0)$ & $\mathrm{n} / \mathrm{a}$ & $173(39.6)$ \\
\hline $45-64$ years & $205(59.6)$ & $130(58.0)$ & $\mathrm{n} / \mathrm{a}$ & $264(60.4)$ \\
\hline $65-74$ years & $\mathrm{n} / \mathrm{a}$ & $\mathrm{n} / \mathrm{a}$ & $116(84.7)$ & $\mathrm{n} / \mathrm{a}$ \\
\hline$\geq 75$ years & $\mathrm{n} / \mathrm{a}$ & $\mathrm{n} / \mathrm{a}$ & $21(15.3)$ & $\mathrm{n} / \mathrm{a}$ \\
\hline \multicolumn{5}{|l|}{ Gender, $n(\%)$} \\
\hline Men & $102(29.7)$ & $85(37.9)$ & $52(38.0)$ & 169 (38.7) \\
\hline Women & $242(70.3)$ & $139(62.1)$ & $85(62.0)$ & $268(61.3)$ \\
\hline \multicolumn{5}{|l|}{ Race, $n(\%)$} \\
\hline White & $263(76.5)$ & $209(93.3)$ & $130(94.9 \%)$ & $413(94.5)$ \\
\hline Black or African American & $20(5.8)$ & $11(4.9)$ & 0 & $18(4.1)$ \\
\hline Other & $29(8.4)$ & 0 & 0 & $1(0.2)$ \\
\hline Not reported & $25(7.3)$ & 0 & $2(1.5)$ & 0 \\
\hline Additional categories $^{\mathrm{a}}$ & $<3 \%$ each category & $<3 \%$ each category & $<3 \%$ each category & $<3 \%$ each category \\
\hline \multicolumn{5}{|l|}{ Region, $n(\%)$} \\
\hline Europe & $86(25.0)$ & $134(59.8)$ & $59(43.1)$ & $298(68.2)$ \\
\hline North America & $156(45.3)$ & $90(40.2)$ & $70(51.1)$ & $111(25.4)$ \\
\hline Other $^{\mathrm{b}}$ & $102(29.7)$ & 0 & $8(5.8)$ & $28(6.4)$ \\
\hline
\end{tabular}

n/a not applicable, $S D$ standard deviation

${ }^{a}$ Additional race categories included Asian, American Indian or Alaskan Native, Multiple, and Unknown

${ }^{\mathrm{b}}$ TRANSFORM-1, TRANSFORM-3, and SUSTAIN-1 also had study sites in Central and South America, and TRANSFORM-3 also had study sites in Africa

\subsubsection{TRANSFORM-3 (Patients $\geq 65$ Years, Flexible-Dosed 4-Week Study, Twice Weekly Intranasal Dosing)}

This study included a lower dose of $28 \mathrm{mg}$ as a starting dose and a dose option in addition to $56 \mathrm{mg}$ and $84 \mathrm{mg}$ after day 1. For UPSIT ${ }^{\circledR}$ scores, there were no remarkable differences observed between patients treated with intranasal esketamine + oral antidepressant and those treated with intranasal placebo + oral antidepressant (Table 2).

\subsubsection{SUSTAIN-1 (Long-Term Maintenance Study, Individualized Dosing Once Week or Every Other Week)}

No clinically important changes in sense of smell as measured by the UPSIT ${ }^{\circledR}$ and the S\&S-T were observed. For direct-entry patients in SUSTAIN-1 who had an open-label IND phase, the mean percent changes in the UPSIT ${ }^{\circledR}$ scores from baseline (IND) in patients receiving intranasal esketamine + oral antidepressant during the open-label IND phase and OP phase were comparable over time. Similarly, for patients transferred into the OP phase of the SUSTAIN-1 study after achieving response in the TRANSFORM-1 or TRANSFORM-2 DB IND phase, the mean percent changes in UPSIT $^{\circledR}$ scores from baseline (IND) in the intranasal esketamine + oral antidepressant and intranasal placebo + oral antidepressant treatment groups were comparable over time in the MA phase.

Furthermore, in SUSTAIN-1, the change from baseline to endpoint in UPSIT $^{\circledR}$ scores was not significantly different across the treatment groups $(p=0.665)$, indicating that esketamine did not alter the ability to identify odors (Table 4 and Supplementary Table 3 [see ESM]). The change from baseline for the S\&S-T scores in the intranasal esketamine + oral antidepressant group were comparable to the changes in the placebo nasal spray and oral antidepressant group in the MA phase (Tables 5, 6). The incidence of an olfactory deficit (smell threshold $\geq-2.40$ ) was $0.7 \%$ (3 of 430 patients) and $1.3 \%$ (6 of 454 ) in patients treated with intranasal esketamine + oral antidepressant during the open-label IND phase and OP phase, respectively. In the MA (randomized withdrawal) 
Table 2 UPSIT $^{\circledR}$ total score means and mean percent changes from baseline over time for patients in induction phases of TRANSFORM-1 [15], TRANSFORM-2 [16], and TRANSFORM-3 [17] studies (safety analysis set)

\begin{tabular}{|c|c|c|c|}
\hline \multirow[t]{3}{*}{ Parameters } & \multicolumn{3}{|c|}{ UPSIT $^{\circledR}$ total score $(\%)$} \\
\hline & \multirow[t]{2}{*}{$N$} & \multirow[t]{2}{*}{ Mean (SD) } & $\begin{array}{l}\text { Percent change } \\
\text { from week } 2 \\
\text { (SC) }\end{array}$ \\
\hline & & & Mean (SD) \\
\hline \multicolumn{4}{|c|}{ TRANSFORM-1 induction phase } \\
\hline \multicolumn{4}{|c|}{ ESK nasal spray $56 \mathrm{mg}+$ oral AD } \\
\hline Week 2 (SC) & 115 & $83.5(8.94)$ & \\
\hline Day 15 & 108 & $85.2(7.67)$ & $2.5(9.96)$ \\
\hline Day 28 & 111 & $84.7(8.22)$ & $1.6(9.12)$ \\
\hline Endpoint (DB) & 113 & $84.5(8.34)$ & $1.5(9.13)$ \\
\hline \multicolumn{4}{|c|}{ ESK nasal spray $84 \mathrm{mg}+$ oral AD } \\
\hline Week 2 (SC) & 116 & $84.0(9.40)$ & \\
\hline Day 15 & 109 & $84.4(10.44)$ & $0.2(9.30)$ \\
\hline Day 28 & 100 & $85.2(9.12)$ & $1.4(9.60)$ \\
\hline Endpoint (DB) & 111 & $85.6(8.97)$ & $1.7(9.29)$ \\
\hline \multicolumn{4}{|c|}{ Placebo nasal spray + oral AD } \\
\hline Week 2 (SC) & 113 & $82.5(11.35)$ & \\
\hline Day 15 & 111 & $83.1(10.19)$ & $1.5(12.23)$ \\
\hline Day 28 & 108 & $83.5(10.13)$ & $2.0(11.98)$ \\
\hline End point (DB) & 112 & $83.4(10.13)$ & $1.7(11.87)$ \\
\hline \multicolumn{4}{|c|}{ TRANSFORM-2 induction phase } \\
\hline \multicolumn{4}{|c|}{ ESK nasal spray + oral AD } \\
\hline Week 2 (SC) & 115 & $83.3(9.46)$ & \\
\hline Day 15 & 111 & $83.5(10.71)$ & $0.3(9.04)$ \\
\hline Day 28 & 104 & $84.1(9.68)$ & $1.4(9.80)$ \\
\hline Endpoint (DB) & 111 & $84.2(9.61)$ & $1.5(9.80)$ \\
\hline \multicolumn{4}{|c|}{ Placebo nasal spray + oral AD } \\
\hline Week 2 (SC) & 109 & $82.3(10.03)$ & \\
\hline Day 15 & 103 & $83.7(10.70)$ & $1.8(8.09)$ \\
\hline Day 28 & 101 & $82.9(10.75)$ & $1.3(10.48)$ \\
\hline Endpoint (DB) & 105 & $83.2(10.71)$ & $1.5(10.36)$ \\
\hline \multicolumn{4}{|c|}{ TRANSFORM-3 induction phase } \\
\hline \multicolumn{4}{|c|}{ ESK nasal spray + oral AD } \\
\hline Week 2 (SC) & 70 & $76.2(13.35)$ & \\
\hline Day 28 & 63 & $72.0(14.44)$ & $-4.1(15.12)$ \\
\hline Endpoint (DB) & 63 & $72.0(14.44)$ & $-4.1(15.12)$ \\
\hline \multicolumn{4}{|c|}{ Placebo nasal spray + oral AD } \\
\hline Week 2 (SC) & 65 & $73.7(15.34)$ & \\
\hline Day 28 & 61 & $72.7(16.41)$ & $0.73(19.59)$ \\
\hline Endpoint (DB) & 61 & $72.7(16.41)$ & $0.73(19.59)$ \\
\hline
\end{tabular}

$\mathrm{UPSIT}^{\circledR}$ total score $(\%)$ is defined as [(\#correct responses)/(\#completed responses) $]^{*} 100$

$A D$ antidepressant, $D B$ double-blind, ESK esketamine, $S C$ screening, $S D$ standard deviation, $U P S I T^{\circledR}$ University of Pennsylvania Smell Identification Test

phase, the incidence of an olfactory deficit was $2.0 \%$ (3 of 152 patients) in those continuing in the intranasal esketamine + oral antidepressant group and 2.1\% (3 of 144 patients) in those switched to the intranasal placebo + oral antidepressant group (Table 4). These low olfactory test scores in these participants were clearly preexisting and were not considered to be due to study interventions. 
Table 3 S\&S-T score means and changes from baseline over time for patients in double-blind induction phase of TRANSFORM-1 [15] and TRANSFORM-2 [16] studies (safety analysis set)

\begin{tabular}{|c|c|c|c|c|}
\hline \multirow[t]{3}{*}{ Parameters } & \multicolumn{4}{|c|}{ Smell threshold } \\
\hline & \multirow[t]{2}{*}{$N$} & \multirow[t]{2}{*}{ Mean (SD) } & \multicolumn{2}{|c|}{ Change from baseline } \\
\hline & & & $N$ & Mean (SD) \\
\hline \multicolumn{5}{|c|}{ TRANSFORM-1 induction phase } \\
\hline \multicolumn{5}{|c|}{ ESK nasal spray $56 \mathrm{mg}+$ oral AD } \\
\hline Week 2 (SC) & 110 & $-5.12(1.390)$ & & \\
\hline Day 28 & 104 & $-5.05(1.295)$ & 101 & $0.03(1.466)$ \\
\hline Endpoint (DB) & 104 & $-5.05(1.295)$ & 101 & $0.03(1.466)$ \\
\hline \multicolumn{5}{|c|}{ ESK nasal spray $84 \mathrm{mg}+$ oral AD } \\
\hline Week 2 (SC) & 114 & $-5.21(1.453)$ & & \\
\hline Day 28 & 100 & $-5.32(1.417)$ & 99 & $-0.09(1.238)$ \\
\hline Endpoint (DB) & 100 & $-5.32(1.417)$ & 99 & $-0.09(1.238)$ \\
\hline \multicolumn{5}{|c|}{ Placebo nasal spray $84 \mathrm{mg}+$ oral AD } \\
\hline Week 2 (SC) & 107 & $-5.27(1.273)$ & & \\
\hline Day 28 & 106 & $-5.25(1.417)$ & 101 & $-0.03(1.256)$ \\
\hline Endpoint (DB) & 106 & $-5.25(1.417)$ & 101 & $-0.03(1.256)$ \\
\hline \multicolumn{5}{|c|}{ TRANSFORM-2 induction phase } \\
\hline \multicolumn{5}{|c|}{ ESK nasal spray + oral AD } \\
\hline Week 2 (SC) & 113 & $-5.56(1.303)$ & & \\
\hline Day 28 & 109 & $-5.68(1.238)$ & 108 & $-0.12(1.356)$ \\
\hline Endpoint (DB) & 109 & $-5.68(1.238)$ & 108 & $-0.12(1.356)$ \\
\hline \multicolumn{5}{|c|}{ Placebo nasal spray + oral AD } \\
\hline Week 2 (SC) & 107 & $-5.48(1.356)$ & & \\
\hline Day 28 & 100 & $-5.65(1.346)$ & 98 & $-0.04(1.170)$ \\
\hline Endpoint (DB) & 100 & $-5.65(1.346)$ & 98 & $-0.04(1.170)$ \\
\hline
\end{tabular}

Smell threshold is the mean of the 4th through 7 th reversals

$A D$ antidepressant, $D B$ double-blind, $E S K$ esketamine, $S C$ screening, $S D$ standard deviation, $S \& S-T$ Snap \& Sniff ${ }^{\circledR}$ Odor Detection Threshold Test

In the MA phase, although S\&S-T threshold values of the esketamine + oral antidepressant group showed a numerical tendency to be lower than those of the placebo + oral antidepressant group (i.e., greater sensitivity), this difference was not statistically significant ( $p=0.150$ ) (Supplementary Table 4 in the ESM).

\subsection{Nasal Tolerability}

\subsubsection{Nasal examination}

Across all four studies, the majority of esketamine-treated patients had no adverse findings on the nasal examination (conducted pre-dose on an intranasal dosing day). A small number of patients reported mild-to-moderate symptoms of nasal discharge, nasal crusting, or nasal erythema (Supplementary Tables 5-9 in the ESM). No findings identified were rated as severe by the investigator. Epistaxis was noted in one patient in the OP phase (esketamine + oral antidepressant treatment group) of SUSTAIN-1.

\subsubsection{Nasal Symptom Questionnaire}

Across these controlled studies, most patients reported no nasal symptoms or mild nasal symptoms for individual items on the questionnaire. Overall, the most frequently reported transient post-dose nasal symptoms of moderate or severe intensity (reported in at least $5 \%$ of patients) were postnasal drip (8.7\%), taste disturbance (7.8\%), and stuffy nose (6.9\%). The most common post-dose nasal symptoms of moderate or severe intensity in esketamine-treated patients were postnasal drip (9.4\%), taste disturbance ( $8.2 \%)$, stuffy nose $(7.7 \%)$, and runny nose $(5.5 \%)$. The most common nasal symptoms in the placebo group were postnatal drip and taste disturbance (6.3\% each). No clinically relevant changes in the objective measures (UPSIT ${ }^{\circledR}$ or S\&S-T) were noted in patients reporting post-dose taste disturbance.

An overview of the post-dose nasal symptoms of moderate to severe intensity is provided in Supplementary Table 10 in the ESM, and as follows: 
Table 4 UPSIT $^{\circledR}$ score means and mean percent changes from baseline over time for patients in MA phase of SUSTAIN-1 study (safety analysis set) [14]

\begin{tabular}{|c|c|c|c|}
\hline \multirow[t]{3}{*}{ Parameters } & \multicolumn{3}{|c|}{ UPSIT $^{\circledR}$ total score $(\%)$} \\
\hline & \multirow[t]{2}{*}{$N$} & \multirow[t]{2}{*}{ Mean (SD) } & $\begin{array}{l}\text { Percent change } \\
\text { from baseline } \\
\text { (IND) }\end{array}$ \\
\hline & & & Mean (SD) \\
\hline \multicolumn{4}{|c|}{ ESK nasal spray + oral AD } \\
\hline Baseline (IND) & 152 & $82.5(9.89)$ & \\
\hline Baseline (MA) & 152 & $82.3(10.66)$ & $0.15(11.11)$ \\
\hline Week 4 (MA) & 146 & $82.4(10.98)$ & $0.06(11.64)$ \\
\hline Week 12 (MA) & 111 & $81.5(10.32)$ & $-0.79(11.92)$ \\
\hline Week 20 (MA) & 84 & $83.0(9.73)$ & $1.01(11.91)$ \\
\hline Week 28 (MA) & 55 & $81.8(10.36)$ & $-0.68(15.03)$ \\
\hline Week 36 (MA) & 31 & $81.0(13.57)$ & $-4.34(16.48)$ \\
\hline Week 44 (MA) & 21 & $79.5(8.68)$ & $-6.29(9.18)$ \\
\hline Week 52 (MA) & 12 & $79.8(8.15)$ & $-8.70(9.08)$ \\
\hline Week 60 (MA) & 10 & $80.0(6.01)$ & $-7.85(7.41)$ \\
\hline Week 68 (MA) & 6 & $77.5(13.60)$ & $-13.22(12.85)$ \\
\hline Week 76 (MA) & 3 & $75.0(10.00)$ & $-13.44(9.00)$ \\
\hline Week 84 (MA) & 2 & $76.3(5.30)$ & $-7.35(10.40)$ \\
\hline Endpoint (MA) & 150 & $81.8(12.04)$ & $-0.38(13.22)$ \\
\hline \multicolumn{4}{|c|}{ Placebo nasal spray + oral AD } \\
\hline Baseline (IND) & 144 & $81.5(9.10)$ & \\
\hline Baseline (MA) & 145 & $82.6(9.49)$ & $1.91(11.14)$ \\
\hline Week 4 (MA) & 141 & $81.7(9.55)$ & $0.70(10.69)$ \\
\hline Week 12 (MA) & 82 & $80.1(11.32)$ & 0.07 (14.49) \\
\hline Week 20 (MA) & 51 & $82.3(10.88)$ & $1.92(10.00)$ \\
\hline Week 28 (MA) & 33 & $81.8(10.43)$ & $0.72(11.79)$ \\
\hline Week 36 (MA) & 13 & $77.1(11.40)$ & $-1.31(15.03)$ \\
\hline Week 44 (MA) & 10 & $78.3(7.17)$ & $3.57(10.54)$ \\
\hline Week 52 (MA) & 5 & $76.5(8.40)$ & $2.94(17.29)$ \\
\hline Week 60 (MA) & 3 & $80.0(2.50)$ & $15.74(14.32)$ \\
\hline Week 68 (MA) & 4 & $85.0(4.56)$ & $16.86(21.41)$ \\
\hline Week 76 (MA) & 2 & $82.5(3.54)$ & $29.38(1.96)$ \\
\hline Endpoint (MA) & 144 & $82.4(9.07)$ & $1.73(11.81)$ \\
\hline
\end{tabular}

UPSIT $^{\circledR}$ total score $(\%)$ is defined as $[(\#$ correct responses $) /(\#$ completed responses) $] \times 100$

$A D$ antidepressant, $E S K$ esketamine, IND induction, $M A$ maintenance, $S D$ standard deviation, $U P S I T^{\circledR}$ University of Pennsylvania Smell Identification Test

TRANSFORM- 1 A total of $6 / 115$ patients in the esketamine $56 \mathrm{mg}+$ oral antidepressant, $7 / 116$ patients in the esketamine $84 \mathrm{mg}+$ oral antidepressant, and 5/113 patients in placebo + oral antidepressant reported at least one severe symptom at a $1 \mathrm{~h}$ post-dose timepoint (taste disturbance, postnasal drip, stuffy nose, sore throat, dryness inside nose, and/or itching inside nose).
Table 5 S\&S-T score means and changes from baseline over time for patients in MA phase of SUSTAIN-1 study (safety analysis set) [14]

\begin{tabular}{|c|c|c|c|c|}
\hline \multirow[t]{3}{*}{ Parameters } & \multicolumn{4}{|c|}{ Smell threshold } \\
\hline & \multirow[t]{2}{*}{$N$} & \multirow[t]{2}{*}{ Mean (SD) } & \multicolumn{2}{|c|}{$\begin{array}{l}\text { Change from } \\
\text { baseline (MA) }\end{array}$} \\
\hline & & & $N$ & Mean (SD) \\
\hline \multicolumn{5}{|c|}{ ESK nasal spray + oral AD } \\
\hline Baseline (IND) & 147 & $-5.64(1.46)$ & & \\
\hline Baseline (MA) & 152 & $-5.75(1.37)$ & 147 & $-0.10(1.25)$ \\
\hline Week 12 (MA) & 139 & $-5.90(1.22)$ & 135 & $-0.25(1.31)$ \\
\hline Week 24 (MA) & 75 & $-5.97(1.37)$ & 74 & $-0.28(1.34)$ \\
\hline Week 36 (MA) & 37 & $-5.93(1.52)$ & 37 & $-0.17(1.72)$ \\
\hline Week 48 (MA) & 18 & $-6.16(1.32)$ & 18 & $-0.54(1.70)$ \\
\hline Week 60 (MA) & 11 & $-6.05(1.05)$ & 11 & $-0.32(1.37)$ \\
\hline Week 72 (MA) & 4 & $-6.88(1.19)$ & 4 & $-1.06(1.25)$ \\
\hline Week 84 (MA) & 2 & $-6.13(0.00)$ & 2 & $-0.81(0.62)$ \\
\hline Endpoint (MA) & 141 & $-5.92(1.25)$ & 137 & $-0.28(1.24)$ \\
\hline \multicolumn{5}{|c|}{ Placebo nasal spray + oral AD } \\
\hline Baseline (IND) & 141 & $-5.73(1.39)$ & & \\
\hline Baseline (MA) & 143 & $-5.84(1.30)$ & 140 & $-0.08(1.36)$ \\
\hline Week 12 (MA) & 136 & $-5.69(1.20)$ & 133 & $0.05(1.30)$ \\
\hline Week 24 (MA) & 47 & $-5.76(1.30)$ & 45 & $0.20(1.35)$ \\
\hline Week 36 (MA) & 19 & $-5.92(1.42)$ & 19 & $-0.16(1.30)$ \\
\hline Week 48 (MA) & 7 & $-5.70(1.76)$ & 7 & $0.52(0.92)$ \\
\hline Week 60 (MA) & 5 & $-6.15(1.80)$ & 5 & $0.13(1.47)$ \\
\hline Week 72 (MA) & 3 & $-5.08(0.92)$ & 3 & $0.79(0.26)$ \\
\hline Endpoint (MA) & 139 & $-5.77(1.14)$ & 136 & $-0.03(1.37)$ \\
\hline
\end{tabular}

Threshold scores are in $\log _{10}$ units

Smell threshold is the mean of the 4th through 7 th reversals

$A D$ antidepressant, $E S K$ esketamine, $I N D$ induction, $M A$ maintenance, $S D$ standard deviation, $S \& S-T$ Snap \& Sniff ${ }^{\circledR}$ Odor Detection Threshold Test

TRANSFORM-2 Two esketamine-treated patients reported a severe symptom at $1 \mathrm{~h}$ post-dose (postnasal drip: $n=1$; postnasal drip and severe taste disturbance: $n=1)$.

TRANSFORM-3 In patients 65 years and older, six patients in the esketamine group reported at least one severe nasal symptom at $1 \mathrm{~h}$ post-dose (stuffy nose, taste disturbance, and runny nose).

SUSTAIN- 1 The incidence of severe nasal symptoms in all phases was low, with the highest rate observed in the OP phase (16/448; 3.6\%; all in esketamine + oral antidepressant group), and lowest rates in the MA phase (esketamine + oral antidepressant group: $0.7 \%$ [1/151 patients], placebo + oral antidepressant group: $1.4 \%$ [2/144 patients]). Cumulatively, the most frequently reported severe nasal symptoms were stuffy nose, postnasal drip, and taste disturbance. None of the reported symptoms led to discontinuation of the intranasal treatment. 
Table 6 S\&S-T score means and changes from baseline over time for patients in IND and OP phases of SUSTAIN-1 study (safety [IND and $\mathrm{OP}]$ analysis set) [14]

\begin{tabular}{lcccc}
\hline Parameters & $N$ & Mean (SD) & $\begin{array}{l}\text { Change from base- } \\
\text { line (IND) }\end{array}$ \\
\cline { 3 - 5 } & & $N$ & Mean (SD) \\
\hline Smell threshold (IND) test score & & & \\
ESK nasal spray + oral AD & & & \\
Baseline (IND) & 420 & $-5.75(1.36)$ & & \\
Day 28 (IND) & 380 & $-5.80(1.29)$ & 370 & $-0.01(1.06)$ \\
Endpoint (IND) & 380 & $-5.80(1.29)$ & 370 & $-0.01(1.06)$ \\
Smell threshold (OP) test score & & & \\
ESK nasal spray + oral AD & & $-5.62(1.43)$ & & \\
Baseline (IND) & 443 & $-5.66(1.34)$ & 442 & $-0.05(1.15)$ \\
Baseline (OP) & 451 & $-5.78(1.30)$ & 416 & $-0.13(1.34)$ \\
Week 12 (OP) & 426 & $-5.77(1.30)$ & 416 & $-0.12(1.34)$ \\
Endpoint (OP) & 426 & -5 & \\
\hline
\end{tabular}

Threshold scores are in $\log _{10}$ units

Smell threshold is the mean of the 4th through 7 th reversals

$A D$ antidepressant, $E S K$ esketamine, IND induction, $O P$ optimization, $S D$ standard deviation, $S \& S-T$ Snap \& Sniff ${ }^{\circledR}$ Odor Detection Threshold Test

\section{Discussion}

This analysis included 1142 patients with TRD from 21 countries from four phase III studies, including three multicenter, randomized, DB short-term efficacy studies [15-17] and a DB, randomized, multicenter relapse prevention study [14]. In aggregate, this analysis demonstrates that the administration of esketamine nasal spray (28 mg, $56 \mathrm{mg}$, and $84 \mathrm{mg}$ ) is well tolerated nasally and does not impact the ability to smell, as measured by stateof-the-art tests of olfactory identification and detection threshold sensitivity. No dose-response relationship was observed between esketamine doses and the olfactory test scores. Moreover, in the three short-term studies, for both the UPSIT ${ }^{\circledR}$ and S\&S-T test scores, there were no significant differences observed between patients in the intranasal esketamine + oral antidepressant groups compared with patients in placebo nasal spray + oral antidepressant groups in the DB IND phases [15-17]. Similar results were evident during the 4-week, open-label IND phase (direct-entry patients), the 12-week OP phase (open-label for direct-entry patients or DB for transfer-entry patients), and the DB MA phase of the SUSTAIN-1 study [14]. Importantly, long-term intermittent dosing in the relapse prevention study did not significantly impact nasal function. These findings are further supported by earlier nonclinical toxicology studies, in which there was no evidence of safety findings observed related to local tolerability of intranasally administered esketamine with daily dosing in rats and dogs. Further post-marketing safety surveillance will continue over time but evidence to date with shortand long-term evaluations does not suggest an adverse impact on olfactory function.

It has been reported that MDD may be associated with decreases in smell function [26], although not all studies have found such decrements [27] and the magnitude of reported differences relative to healthy controls generally has been $<5 \%$. Of the olfactory tests that have been performed (e.g., odor identification, threshold detection, and discrimination), thresholds appear to be most affected. For example, in one review [28], four of eight threshold studies $(50 \%)$ noted a significant depression-related decrement, four of twelve (33.3\%) studies reported a decrement in odor identification ability, and only one in four $(25 \%)$ reported a decrement in discrimination ability. It is not known whether or to what degree these differences reflect differential sensitivities of such tests or factors that may nonspecifically affect test performance (e.g., reduced attention or effort in depressed persons).

An evaluation of the potential influence of depression on olfactory function was not possible in the current study, as the clinical trials [14-17] did not include participants without MDD, thereby precluding a direct determination of the effects of depression. However, in the current analysis, the average olfactory test scores of this group of patients with TRD fell within or very near the general normal limits expected for non-depressed persons of their age, gender, and nationality $[26,27]$. Indeed, the detection threshold values noted in Tables 5 and 6 fell between the 50th and 60th percentiles of those of non-depressed normal controls [21]. Whether this reflects lack of an effect of MDD/TRD on olfactory test measures or the impact of the antidepressant medications is not clear. The latter is quite likely, however, since antidepressants have been shown to normalize olfactory function in depressed patients [29, 30].

Other notable findings of this study, based on the NSQ results, include evidence that the vast majority of patients did not report any clinically noteworthy adverse nasal symptoms following repeated dosing with esketamine nasal spray. Moreover, the majority of patients reported no nasal symptoms or mild symptoms for individual items on the NSQ. In the short-term studies (TRANSFORM-1, -2, and -3) [15-17], no clinically noteworthy findings were reported on nasal examination, and any abnormalities observed were reported as mild or moderate in severity. No additional safety concerns were observed during repeated nasal examination or NSQ administration during long-term treatment in SUSTAIN-1 [14].

As expected, age was inversely related to the olfactory test scores in the various study groups, in accordance with the well-established adverse influence of age on not only 
tests of odor identification [18] and detection threshold sensitivity $[21,31]$, but on a wide range of other types of olfactory tests [32]. Based on olfactory test scores, there was no interaction observed between age and dose or the duration of intermittent nasal spray drug administration.

Several strengths and limitations of the study design merit comment. Among its strengths are its large cohort of patients with TRD, randomized DB assessments in multiple clinics across many regions and countries, and longitudinal assessments. Other strengths include stateof-the-art testing of both odor identification and detection threshold function, and multiple measures of nasal tolerance and nasal health over time. One limitation (which is not considered relevant to the key findings of no negative impact of esketamine on nasal function), was the aforementioned lack of an unmedicated, non-depressed control group. In this work, all patients had TRD and were receiving open-label oral antidepressants started simultaneously with the intranasally administered esketamine or nasal placebo. Thus, it is not possible to discern, from these data, the specific effects of MDD on smell function independent of the effects of baseline oral antidepressant medication on nasal function. Another limitation of the study is that, in an effort to reduce burden on patients, only short-term olfactory data were collected for older patients ( $\geq 65$ years) and this was performed with three UPSIT ${ }^{\circledR}$ booklets, instead of four. The generalizability of the study findings is limited by the exclusion of patients with significant psychiatric or medical co-morbidities or moderate/ severe substance use disorder, with a greater proportion of female to male patients, consistent with the demographic characteristics of the patients' population.

\section{Conclusion}

Based on comprehensive testing in short-term (4 weeks) and long-term (16-100 weeks) studies in patients with TRD, repeated intermittent administration of esketamine nasal spray (twice weekly, once weekly, or once every other week) did not demonstrate evidence of adverse effects on the ability to smell or on key elements of nasal function.

Supplementary Information The online version contains supplementary material available at https://doi.org/10.1007/s40263-021-00826-9.

Acknowledgements Himabindu Gutha, Ph.D. and Vaibhav Deshpande, $\mathrm{Ph} . \mathrm{D}$. (SIRO Clinpharm Pvt. Ltd., India) provided writing assistance, which was funded by Janssen Research \& Development, LLC. Ellen Baum, Ph.D. (Janssen Global Services, LLC) provided additional editorial support for this manuscript. Authors thank the study participants without whom this study would not have been accomplished, and the investigational site staff for their contribution in this study.

\section{Declarations}

Funding The studies presented in this report were supported by Janssen Research \& Development, LLC, USA. Open access funding was provided by Janssen Research \& Development, LLC, USA.

Conflict of Interest All the authors, except Dr. Doty, Ms. Wylie and Dr. Singh, are employees of Janssen Research \& Development, LLC and shareholders of Johnson \& Johnson. Dr. Doty is President and major shareholder of Sensonics International, the manufacturer and distributor of smell and taste tests, including the tests used in these studies. He is a consultant to Acorda Therapeutics, Eisai Co., Ltd., Merck Pharmaceuticals, The Michael J. Fox Foundation for Parkinson's Research, and Johnsons \& Johnson. He receives royalties from Cambridge University Press, Johns Hopkins University Press, and John Wiley \& Sons, Inc. Ms Wylie was employed at Sensonics International and the Smell and Taste Center at the Hospital of University of Pennsylvania when this study was conducted. Jaskaran Singh worked on the clinical development program of esketamine for treatment-resistant depression during his employment by Janssen Research \& Development, LLC; he is currently employed by Neurocrine Biosciences, San Diego, CA.

Ethics Approval The protocol and informed consent forms were approved by local independent ethics committees/Institutional Review Boards. All procedures performed in studies involving human participants were in accordance with the International Council for Harmonisation Good Clinical Practice Consolidated Guideline, the applicable local laws and regulatory requirements of the countries in which the trial was conducted, and with the 1964 Helsinki declaration and its later amendments or comparable ethical standards.

Consent to Participate Written informed consent/assent was obtained from all individual patients included in the trial (or their parent/legal representative) before any trial-specific procedures were performed or any patient-related data were collected.

Consent for Publication Not applicable.

Availability of Data and Material All data generated or analyzed during this study are included in this published article and its supplementary information files. The data sharing policy of Janssen Pharmaceutical Companies of Johnson \& Johnson is available at https://www.janss en.com/clinical-trials/transparency. As noted on this site, requests for access to the study data can be submitted through Yale Open Data Access [YODA] Project site at http://yoda.yale.edu.

Author Contributions Conception and design: MF, VP, ED, JS, RO-R. Collection and assembly of data: MD and CW, MF, VP. Data analysis and interpretation: RLD. All authors participated in supervising recruitment, monitoring of data quality, and development and review of this manuscript and confirm that they have read the journal's position on issues involved in ethical publication and affirm that this report is consistent with those guidelines. All authors meet ICMJE criteria and all those who fulfilled those criteria are listed as authors. All authors had access to the study data, provided direction and comments on the manuscript, made the final decision about where to publish these data and approved submission to this journal.

Open Access This article is licensed under a Creative Commons Attribution-NonCommercial 4.0 International License, which permits any non-commercial use, sharing, adaptation, distribution and reproduction 
in any medium or format, as long as you give appropriate credit to the original author(s) and the source, provide a link to the Creative Commons licence, and indicate if changes were made. The images or other third party material in this article are included in the article's Creative Commons licence, unless indicated otherwise in a credit line to the material. If material is not included in the article's Creative Commons licence and your intended use is not permitted by statutory regulation or exceeds the permitted use, you will need to obtain permission directly from the copyright holder. To view a copy of this licence, visit http://creativecommons.org/licenses/by-nc/4.0/.

\section{References}

1. Al-Harbi KS. Treatment-resistant depression: therapeutic trends, challenges, and future directions. Patient Prefer Adherence. 2012;6:369-88.

2. Fava M, Davidson KG. Definition and epidemiology of treatment-resistant depression. Psychiatr Clin North Am. 1996;19(2):179-200.

3. Berlim MT, Turecki G. Definition, assessment, and staging of treatment-resistant refractory major depression: a review of current concepts and methods. Can J Psychiatry. 2007;52(1):46-54.

4. Daly EJ, Singh JB, Fedgchin M, Cooper K, Lim P, Shelton RC, et al. Efficacy and safety of intranasal esketamine adjunctive to oral antidepressant therapy in treatment-resistant depression: a randomized clinical trial. JAMA Psychiat. 2018;75(2):139-48.

5. Wei Y, Chang L, Hashimoto K. A historical review of antidepressant effects of ketamine and its enantiomers. Pharmacol Biochem Behav. 2020;190:172870.

6. Esketamine (Spravato ${ }^{\mathrm{TM}}$ ) Nasal Spray Prescribing Information. Titusville, NJ: Janssen Pharmaceuticals, Inc. 2019. https://www. accessdata.fda.gov/drugsatfda_docs/label/2019/211243lbl.pdf. Accessed 1 Dec 2020.

7. Andrade C. Intranasal drug delivery in neuropsychiatry: focus on intranasal ketamine for refractory depression. J Clin Psychiatry. 2015;76(5):e628-31.

8. Genter MB, Krishan M, Prediger RD. The olfactory system as a route of delivery for agents to the brain and circulation. In: Doty RL, editor. Handbook of olfaction and gustation. 3rd edn. WileyBlackwell; 2015. pp. 453-84.

9. Fanta S, Kinnunen M, Backman JT, Kalso E. Population pharmacokinetics of S-ketamine and norketamine in healthy volunteers after intravenous and oral dosing. Eur J Clin Pharmacol. 2015;71(4):441-7.

10. Food and Drug Administration (FDA). Center for Drug Evaluation and Research. Nacran nasal spray medical review comments. 2015. https://www.accessdata.fda.gov/drugsatfda_docs/nda/2015/ 208411Orig1s000MedR.pdf. Accessed 1 Dec 2020.

11. Calderón-Garcidueñas L, González-Maciel A, Reynoso-Robles R, Kulesza RJ, Mukherjee PS, Torres-Jardón R, et al. Alzheimer's disease and alpha-synuclein pathology in the olfactory bulbs of infants, children, teens and adults $\leq 40$ years in Metropolitan Mexico City. APOE4 carriers at higher risk of suicide accelerate their olfactory bulb pathology. Environ Res. 2018;166:348-62.

12. Kuehn BM. Zicam update. JAMA. 2010;303(16):1587. https:// doi.org/10.1001/jama.2010.457.

13. Doty RL, Shaman P, Dann M. Development of the University of Pennsylvania Smell Identification Test: a standardized microencapsulated test of olfactory function. Physiol Behav. 1984;32:489-502.
14. Daly EJ, Trivedi MH, Janik A, Li H, Zhang Y, Li X, et al. Efficacy of esketamine nasal spray plus oral antidepressant treatment for relapse prevention in patients with treatment-resistant depression: a randomized clinical trial. JAMA Psychiat. 2019;76(9):893-903.

15. Fedgchin M, Trivedi M, Daly EJ, Melkote R, Lane R, Lim P, et al. Efficacy and safety of fixed-dose esketamine nasal spray combined with a new oral antidepressant in treatment-resistant depression: results of a randomized, double-blind, active-controlled study (TRANSFORM-1). Int J Neuropsychopharmacol. 2019;22(10):616-30.

16. Popova V, Daly EJ, Trivedi M, Cooper K, Lane R, Lim P, et al. Efficacy and safety of flexibly dosed esketamine nasal spray combined with a newly initiated oral antidepressant in treatmentresistant depression: a randomized double-blind active-controlled study. Am J Psychiatry. 2019;176(6):428-38.

17. Ochs-Ross R, Daly EJ, Zhang Y, Lane R, Lim P, Morrison RL, et al. Efficacy and safety of esketamine nasal spray plus an oral antidepressant in elderly patients with treatment-resistant depression-TRANSFORM-3. Am J Geriatr Psychiatry. 2020;28(2):121-41.

18. Doty RL, Shaman P, Applebaum SL, Giberson R, Siksorski L, Rosenberg L. Smell identification ability: changes with age. Science. 1984;226(4681):1441-3.

19. Doty RL, Wylie C, Potter M, Beston R, Cope B, Majam K. Clinical validation of the olfactory detection threshold module of the Snap \& Sniff ${ }^{\circledR}$ olfactory test system. Int Forum Allergy Rhinol. 2019;9(9):986-92.

20. Doty RL. The Smell Identification Test ${ }^{\mathrm{TM}}$ Administration Manual. 3rd edition. Haddon Heights, NJ: Sensonics; 1995.

21. Doty RL. The Snap \& Sniff ${ }^{\circledR}$ Olfactory Test System: Threshold Administration Manual. Haddon Heights, NJ: Sensonics; 2020.

22. Doty RL, Frye RE, Agrawal U. Internal consistency reliability of the fractionated and whole University of Pennsylvania Smell Identification Test. Percept Psychophys. 1989;45(5):381-4.

23. Schwartz BS, Doty RL, Monroe C, Frye R, Barker S. Olfactory function in chemical workers exposed to acrylate and methacrylate vapors. Am J Pub Health. 1989;79(5):613-8.

24. Yilmaz Y, Karakas Z, Uzun B, Sen C, Comoglu S, Orhan KS, et al. Olfactory dysfunction and quality of life in patients with transfusion-dependent thalassemia. Eur Arch Otorhinolaryngol. 2017;274(9):3417-21.

25. Hawkes CH, Doty RL. Smell and taste disorders. Cambridge: Cambridge University Press; 2018.

26. Kamath V, Paksarian D, Cui L, Moberg PJ, Turetsky BI, Merikangas KR. Olfactory processing in bipolar disorder, major depression, and anxiety. Bipolar Disord. 2018;20(6):547-55.

27. Amsterdam JD, Settle RG, Doty RL, Abelman E, Winokur A. Taste and smell perception in depression. Biol Psychiatry. 1987;22(12):1481-5.

28. Taalman H, Wallace C, Milev R. Olfactory functioning and depression: a systematic review. Front Psychiatry. 2017;8:190.

29. Croy I, Symmank A, Schellong J, Hummel C, Gerber J, Joraschky $\mathrm{P}$, et al. Olfaction as a marker for depression in humans. J Affect Disord. 2014;160:80-6.

30. Pause BM, Raack N, Sojka B, Göder R, Aldenhoff JB, Ferstl R. Convergent and divergent effects of odors and emotions in depression. Psychophysiology. 2003;40(2):209-25.

31. Deems DA, Doty RL. Age-related changes in the phenyl ethyl alcohol odor detection threshold. Trans Pa Acad Ophthalmol Otolaryngol. 1987;39(1):646-50.

32. Doty RL, Kamath V. The influences of age on olfaction: a review. Front Psychol. 2014;5:20. 


\section{Authors and Affiliations}

Richard L. Doty ${ }^{1}$. Vanina Popova ${ }^{2}$ (D) Crystal Wylie ${ }^{1} \cdot$ Maggie Fedgchin $^{3} \cdot$ Ella Daly $^{4} \cdot$ Adam Janik $^{5}$.

Rachel Ochs-Ross ${ }^{3} \cdot$ Rosanne Lane $^{3} \cdot$ Pilar Lim $^{3} \cdot$ Kim Cooper $^{6} \cdot$ Rama Melkote $^{3} \cdot$ Carol Jamieson $^{7} \cdot$ Jaskaran Singh $^{5,8}$. Wayne C. Drevets ${ }^{5}$

\section{Vanina Popova}

vpopova@its.jnj.com

1 Smell and Taste Center, Department

of Otorhinolaryngology: Head and Neck Surgery,

Perelman School of Medicine, University of Pennsylvania, Philadelphia, PA, USA

2 Janssen Research \& Development, Turnhoutseweg 30, 2340 Beerse, BE, Belgium

3 Janssen Research \& Development, LLC, Titusville, NJ, USA

4 Janssen Scientific Affairs, LLC, Titusville, NJ, USA
5 Janssen Research \& Development, LLC, San Diego, CA, USA

6 Janssen Research \& Development, LLC, Spring House, PA, USA

7 Janssen Research \& Development, LLC, Milpitas, CA, USA

8 Present Address: Neurocrine Biosciences, San Diego, CA, USA 